\title{
A novel class of selective CK2 inhibitors targeting its open hinge conformation
}

Andrea Dalle Vedove ${ }^{1^{*}}$, Francesca Zonta ${ }^{2 *}$, Enrico Zanforlin ${ }^{3}$, Nicola Demitri $^{4}$, Giovanni Ribaudo ${ }^{5}$, Giulia Cazzanelli ${ }^{1}$, Alberto Ongaro ${ }^{3}$, Stefania Sarno ${ }^{2}$, Giuseppe Zagotto ${ }^{3}$, Roberto Battistutta ${ }^{6}$, Maria Ruzzene ${ }^{2}$, Graziano Lolli ${ }^{1}$

Author Affiliations:

${ }^{1}$ Department of Cellular and Computational Biology - CIBIO, University of Trento, Via Sommarive 9, 38123 Trento, Italy ${ }^{2}$ Department of Biomedical Sciences and CNR Institute of Neuroscience, University of Padua, Via U. Bassi 58/B, 35131 Padua, Italy

${ }^{3}$ Department of Pharmaceutical and Pharmacological Sciences, University of Padua, Via F. Marzolo 5, 35131 Padua, Italy ${ }^{4}$ Elettra-Sincrotrone Trieste, S.S. 14 Km 163.5 in Area Science Park, 34149 Basovizza-Trieste, Italy

${ }^{5}$ Department of Molecular and Translational Medicine, Division of Pharmacology, University of Brescia, Brescia, Italy ${ }^{6}$ Department of Chemical Sciences, University of Padua and Institute of Biomolecular Chemistry, National Research Council (CNR), Via Marzolo 1, 35131 Padua, Italy

* The authors contributed equally to the work

Correspondence to Giuseppe Zagotto: giuseppe.zagotto@unipd.it, Roberto Battistutta: roberto.battistutta@unipd.it, Maria Ruzzene: maria.ruzzene@unipd.it and Graziano Lolli: graziano.lolli@unitn.it.

\begin{abstract}
Protein kinase CK2 sustains cancer growth, especially in hematological malignancies. Its inhibitor SRPIN803, based on a 6-methylene-5-imino-1,3,4-thiadiazolopyrimidin-7-one scaffold, showed notable specificity. Our synthesis of SRPIN803 resulted in its constitutional isomer compound 1, where the 2-cyano-2-propenamide group does not cyclise and fuse to the thiadiazole ring. Its crystallographic structure in complex with CK2 $\alpha$ identifies the structural determinants of the reported specificity. Compound 1 explores the CK2 open hinge conformation, extremely rare among kinases, also interacting with side chains from this region. Its optimization lead to the more potent compound 4, which inhibits endocellular CK2, significantly affects viability of tumour cells and shows remarkable selectivity on a panel of 320 kinases.
\end{abstract}




\section{INTRODUCTION}

CK2 is a ubiquitous Ser/Thr protein kinase acting on several substrates whose phosphorylation is involved in a wide range of cellular processes, spanning from cell differentiation to apoptosis [1]. It is a tetramer, formed by two catalytic $\alpha$ and two regulatory $\beta$ subunits. Historically considered a constitutively active kinase [2], it has been proposed that CK2 is instead kept in a non-active state by a peculiar inhibition mechanism, where the tetramers supramolecularly organize into trimers and other more complex structures, and then revert to the active state upon necessity [3-5].

CK2 is expressed at abnormally high levels in various cancers and hematological malignancies [6-7]. Its increased activity has been related with a wide range of responses, such as augmented neovascularisation [8], multidrug resistance [9] and reduced apoptosis [10], all traits that contribute to increased tumour aggressiveness and poorer overall prognosis.

Consistently with the protective role of CK2, tumour cells undergo apoptosis if its catalytic activity is reduced [11]. Furthermore, treatment with CK2 inhibitors induces apoptosis more efficiently in tumour cells than in healthy control cells [12]. Drug discovery efforts devoted at interfering with CK2 activity culminated in the development of CX-4945 [13]; CX-4945 (silmitasertib) has recently been designated as orphan drug for the treatment of cholangiocarcinoma [14] and is in clinical trials for the treatment of various cancers [15].

Potency and selectivity of CK2 inhibitors rely on their interactions with both the hinge region, on one side of the kinase ATP-binding pocket, and the peculiar positively charged region, on the other side. Here we focus on a new class of CK2 inhibitors, based on a central 2-cyano-2-propenamide scaffold variously substituted on both sides to optimize interactions with the two CK2 regions named above. We first determined the crystal structure of CK2 $\alpha$ in complex with SRPIN803, which contains a 6-methylene-5-imino-1,3,4-thiadiazolopyrimidin-7-one scaffold where the 2-cyano-2propenamide is cyclised and fused to a thiadiazole ring (Fig. 1a). SRPIN803 was previously identified 
in a drug development campaign against SRPK1 and kindly provided to us by the authors of this study [16]. SRPK1 is a protein kinase involved in the regulation of various RNA-processing pathways including RNA translation, stability and alternative splicing [17]. SRPIN803, tested against a panel of 306 kinases, showed stronger inhibition of CK2 than of its original target SRPK1, with IC 50 values of $203 \mathrm{nM}$ and $2.4 \mu \mathrm{M}$, respectively, and much lower activity on all other kinases tested [16]. Due to the involvement of SRPK1 in angiogenesis [18] and of CK2 in neovascularisation [8], the combined inhibitory effect of SRPIN803 has been successfully used in the treatment of a mouse model of agerelated macular degeneration, a progressive degenerative condition resulting in vision loss [16]. Unexpectedly, the structure of SRPRIN803, as determined by crystallography in the complex with CK2 $\alpha$, was in the open form as shown in Fig. $1 b$ (i.e., different from the closed form, where the thiadiazole nitrogen reacted with the nitrile carbon, as reported in [16] and in Fig. 1a). SRPIN803 was de novo synthesized in our laboratory according to the published protocol [16] and the product obtained fully characterized, as described below, and named compound 1 (confirming the open form, Fig. 1b). With the correct structure elucidated for the parent inhibitor, we initiated its optimization by producing and characterizing various derivatives of compound $\mathbf{1}$ with increased inhibitory potency against CK2. High-resolution crystallographic structures of CK2 $\alpha$ in complex with the various inhibitors presented here showed interactions with side chains of the CK $2 \alpha$ hinge region. This unique feature among the CK2 inhibitors developed so far, all interacting with main chain atoms of the hinge region, constitutes the basis of the noticeable selectivity of this series of compounds. Moreover, their binding is only compatible with the open hinge conformation, extremely rare among protein kinases [19]. The best inhibitor (compound 4) has $I C_{50}=280 \mathrm{nM}$ against $\mathrm{CK} 2 \alpha$ and only inhibits this kinase and its paralogue $\mathrm{CK} 2 \alpha^{\prime}$ by more than $50 \%$ when tested at $1 \mu \mathrm{M}$ on a panel of 320 kinases. The most promising compounds, when tested on a cell-based assay, demonstrated to be cell permeable and to inhibit endocellular CK2, thus promoting tumor cells death. 


\section{RESULTS AND DISCUSSION}

\section{Synthesis and characterization of compound 1}

SRPIN803 was soaked into CK2 apo crystal and the crystallographic structure of the complex determined at $1.5 \AA ̊$ resolution. Clear positive electron density was present in the kinase ATP pocket, not compatible with the structure of SRPIN803 (Fig. 1a) but instead corresponding to compound 1 (Fig. 1b and S1). The observed electron density did not change when only the first diffraction images were integrated, excluding a dose-dependent X-ray radiation damage. To confirm the X-ray evidence, we de novo synthesized the compound in agreement with the published method for SRPIN803 [16] and fully characterized it. SRPIN803 (Fig. 1a) and compound 1 (Fig. 1b) are constitutional isomers and cannot be distinguished by mass spectrometry. Also, the NMR spectra for compound 1 confirm what reported for SRPIN803 [16] and are compatible with both chemical structures since the amidic proton of compound 1 cannot be distinguished from the imidic proton of SRPIN803 and even the quaternary carbon of the cyano group in compound $\mathbf{1}$ from the same imidic carbon in SRPIN803. However, the IR spectrum of SRPIN803 (Supplementary Information, SI) shows a peak at $2222.16 \mathrm{~cm}^{-1}$ corresponding to the cyano group (the same peak is present in the IR spectra of compounds $\mathbf{2}-\mathbf{1 1}$, Table 1 and SI) and the HPLC profile excluded a mixture of the open (compound 1) and closed (SRPIN803) forms. Finally, and to fully exclude instability of SRPIN803 in the soaking conditions, the crystallographic structure of the isolated compound $\mathbf{1}$ (SI, Fig. S3-S6 and Table S2) confirmed the structure as the one observed in complex with CK2. Extremely similar cases of i) a synthesis aiming at producing a thiadiazolopyrimidinone and resulting in a monocyclic thiadiazolocyanoacrylamide and ii) an incorrect assignment of the chemical structure of a compound in a commercial library have already been reported [20-21]. 


\section{Binding mode of compound 1}

The ATP binding pocket of CK2 can roughly be divided into three areas: the basic region, where Lys68 holds in place the ATP $\beta$-phosphate; the hinge region, on the opposite side, corresponding to the 114-120 stretch of amino acids connecting the N-terminal and C-terminal lobes of the protein; the hydrophobic area, in between the two previous regions, comprising residues Val53, Val66, Ile95, Phe113, Met163 and Ile174 and sandwiching the adenosine moiety of ATP.

Compound 1 is fully buried in the ATP binding pocket of CK2 $\alpha$, spanning from the basic to the hinge region (Fig. 1c). The molecule is anchored to the protein through its guaiacol moiety, with both oxygen atoms tightly interacting with the positively charged ammonium tail of Lys68 side chain. In addition, the guaiacol hydroxyl further interacts with the main chain amide of Asp175 and with a conserved water molecule (W1) [22] that is held in position by Trp176 main chain amide nitrogen and Glu81 side chain (Fig. 1d).

a
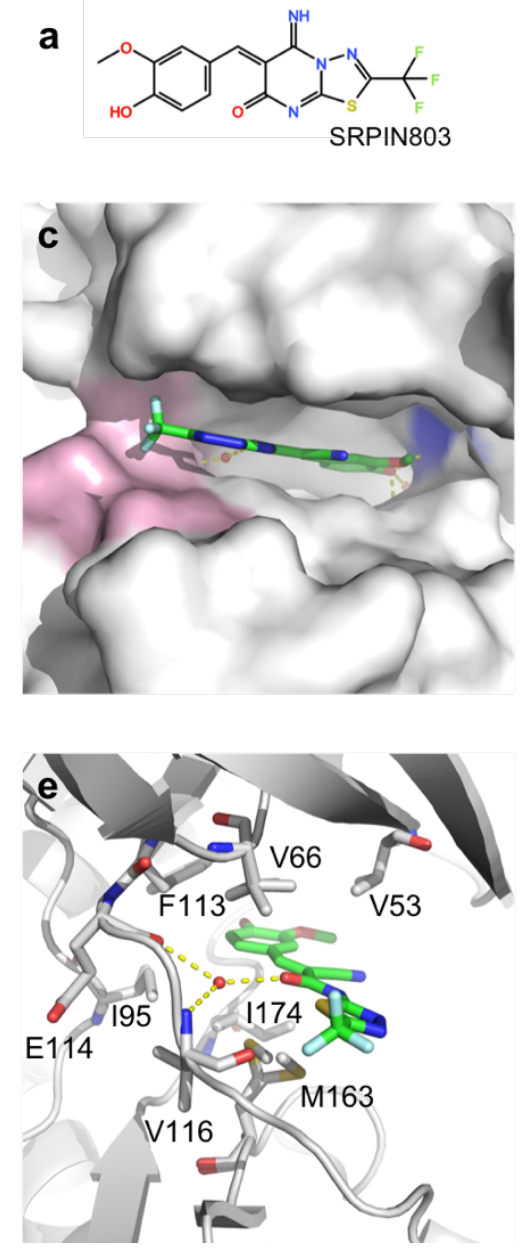
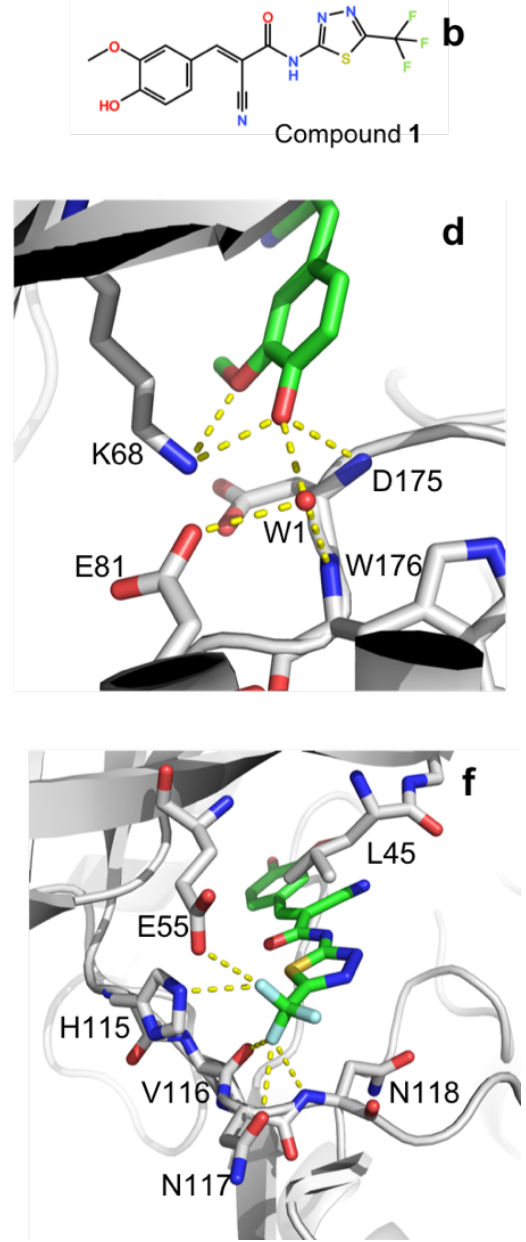
Figure 1. Binding mode of compound 1 in the CK2 ATP-pocket. a) chemical structure of SRPIN803. b) chemical structure of compound 1. c) Compound 1 inserts deeply into the pocket contacting both the basic region (blue surface) and the hinge region (pink surface). d) The guaiacol headgroup is involved in a number of polar interactions with residues in the CK2 basic region. e) The central 2-cyano-2-propenamide group is sandwiched by hydrophobic residues and contacts the hinge region through water-bridged hydrogen bonds. f) the thiadiazole ring stacks in between side chains of Leu45 and Asn118, while the trifluoromethyl end is involved in various polar interactions.

The central 2-cyano-2-propenamide region is involved in Van der Waals and hydrophobic contacts with the aforementioned apolar residues lining the cavity (Fig. 1e). It also contacts the hinge region through a water-mediated hydrogen bond between the propenamide carbonyl oxygen and Glu114 main chain oxygen and Val116 main chain nitrogen. The nitrile group also contributes to the binding affinity through its electron-withdrawal effect, with the ultimate increase in acidity of the guaiacol hydroxyl; its pKa is estimated in 8.8 with a decrease of about 1 unit with respect to molecules with a methyl or an ethynyl group in place of the nitrile [23]. In the local context of the CK2 basic region, the deprotonated form of the inhibitor should be, at least minimally, populated.

Finally, the thiadiazole ring is sandwiched between side chains of Leu45 and Asn118, while the trifluoromethyl group gets jammed in between the hinge region and the $\mathrm{N}$-terminal lobe (Fig. 1f). Several polar interactions are established between two fluorine atoms and the protein matrix, namely with Val116 main chain oxygen, Asn118 main chain nitrogen and the side chains of Glu55, Asn117 and His115, although poorly oriented for the establishment of hydrogen and halogen bonds. The observed binding mode of compound $\mathbf{1}$ in CK2 is different from the proposed docking pose of SRPIN803 in complex with the same kinase (Fig. S2).

\section{Structure-activity relationship for 2-cyano-2-propenamide compounds}

$\mathrm{IC}_{50}$ for compound 1 was estimated in $1.37 \mu \mathrm{M}$ against $\mathrm{CK} 2 \alpha$. In order to increase the affinity of compound 1, we focused on the two regions flanking the central 2-cyano-2-propenamide scaffold (Table 1 and Scheme 1). 


\section{Scheme 1}

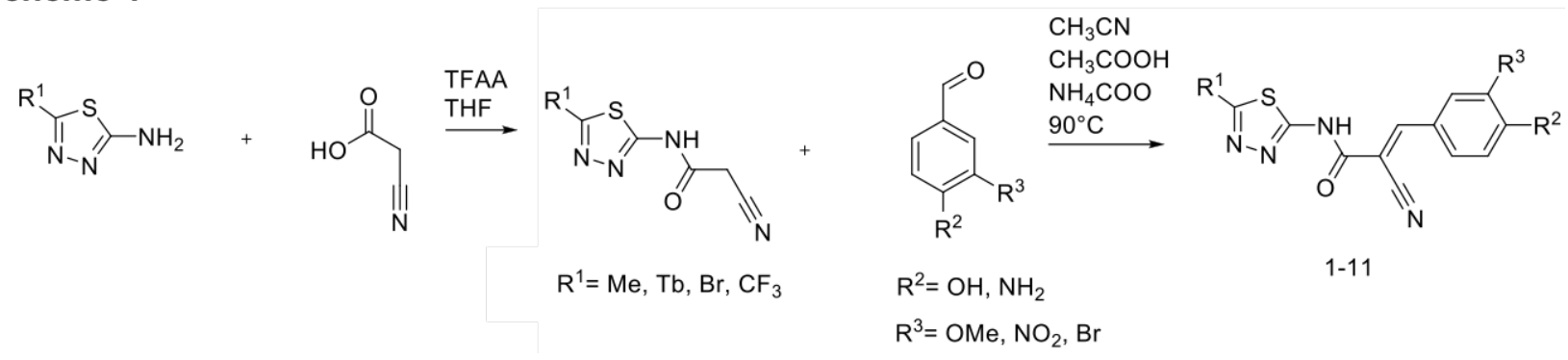

First, compounds 2-4 were synthesized as derivatives of compound $\mathbf{1}$ with the trifluoromethyl group substituted with methyl, tert-butyl and bromine, respectively. Compound $\mathbf{2}$ is only slightly more potent than $\mathbf{1}$ while compound $\mathbf{3}$ shows a fourfold increase in inhibitor potency with respect to compound 1 ; compound 4 is the most potent among all tested inhibitors in this work with an $\mathrm{IC}_{50}=$ $280 \mathrm{nM}$.

The position of the guaiacol group is almost identical in compounds $\mathbf{1}, \mathbf{3}$ and $\mathbf{4}$ (Fig. 2a) in complex with $\mathrm{CK} 2 \alpha$, as determined by X-ray crystallography; all its interactions with the protein matrix are conserved. In compound 3, the bulkier tert-butyl-thiadiazole group moves significantly toward the C-terminal part of the hinge region, as compared with $\mathbf{1}$, to optimize interactions with residues from both the hinge and the CK2 N-terminal lobe. The bromo-thiadiazole tail of compound 4 is closer to the hinge region with respect to compound $\mathbf{3}$, but also to compound $\mathbf{1}$ (Fig. 2a); an interaction between the thiadiazole sulphur and Val116 main chain oxygen is established. The S--O distance decreases to $3.1 \AA\left(\mathrm{C}_{\mathrm{ar}} \mathrm{S}-\mathrm{S}-\mathrm{O}\right.$ angle $\left.127^{\circ}\right)$, being $3.5 \AA$ in the complex with compound 1 . The thiadiazole sulfur acts as an electrophile due to the donation of its $p_{z}$ electrons to the aromatic system and interacts with the carbonyl oxygen through $\sigma$-hole bonding [24]. Strength of interaction increases with the number of the electron-withdrawing nitrogen atoms on the ring, so that thiadiazole is much favoured over thiazole and thiophene. Energy for the thiadiazole S--O interaction, stronger than a typical hydrogen bond, has been computed in $-27 \mathrm{~kJ} / \mathrm{mol}$ at the optimal distance (3 $\AA$ ) and angle $\left(156^{\circ}\right)$ [24]. 
On the opposite side of the molecule, the guaiacol methoxyl was then replaced by a nitro group with the aim of increasing the acidity of the ortho hydroxyl. The predicted effect is a large decrease of the pKa to a value of 5.7 with the outcome of a fully deprotonated phenolate in the basic region of the CK2 pocket [23]. The o-nitrophenolic compounds 5-7 were synthesised, which included a thiadiazole ring derivatised with either trifluoromethyl or methyl or bromine, respectively. Inhibitory power, with respect to compound 1, was doubled for compound $\mathbf{5}$ and the improvement was almost three times for compound $\mathbf{6}$, as compared with $\mathbf{2}$. This confirmed the favourable contribution of the o-nitro group for these compounds. Interestingly, this contribution is abolished in the context of the bromo-thiadiazole compounds and the 0 -nitrophenolic compound 7 performed slightly worse than the guaiacol-bearing compound 4.

The crystallographic structure of compound 7 in complex with CK2 $\alpha$ shows a binding mode very similar to that observed for compound 4, with a limited sliding towards the hinge region to accommodate the bulkier (with respect to the methoxyl) nitro group in the CK2 basic region (Fig. 2b). The $o$-nitrophenolic group maintains all interactions observed for the guaiacol ring, with Lys68 side chain involved in a salt bridge to the nitro group (Fig. 1d and 2c). The nitro group, however, gets also close to the Asp175 side chain. The thiadiazole sulphur is at $3.1 \AA\left(\mathrm{C}_{\mathrm{ar}}-\mathrm{S}-\mathrm{-O}\right.$ angle $\left.133^{\circ}\right)$ from Val116 carbonyl oxygen. The slight shift of compound $\mathbf{7}$ with respect to 4 causes a larger protrusion of the bromine atom toward the hinge region (Fig. 2b). 

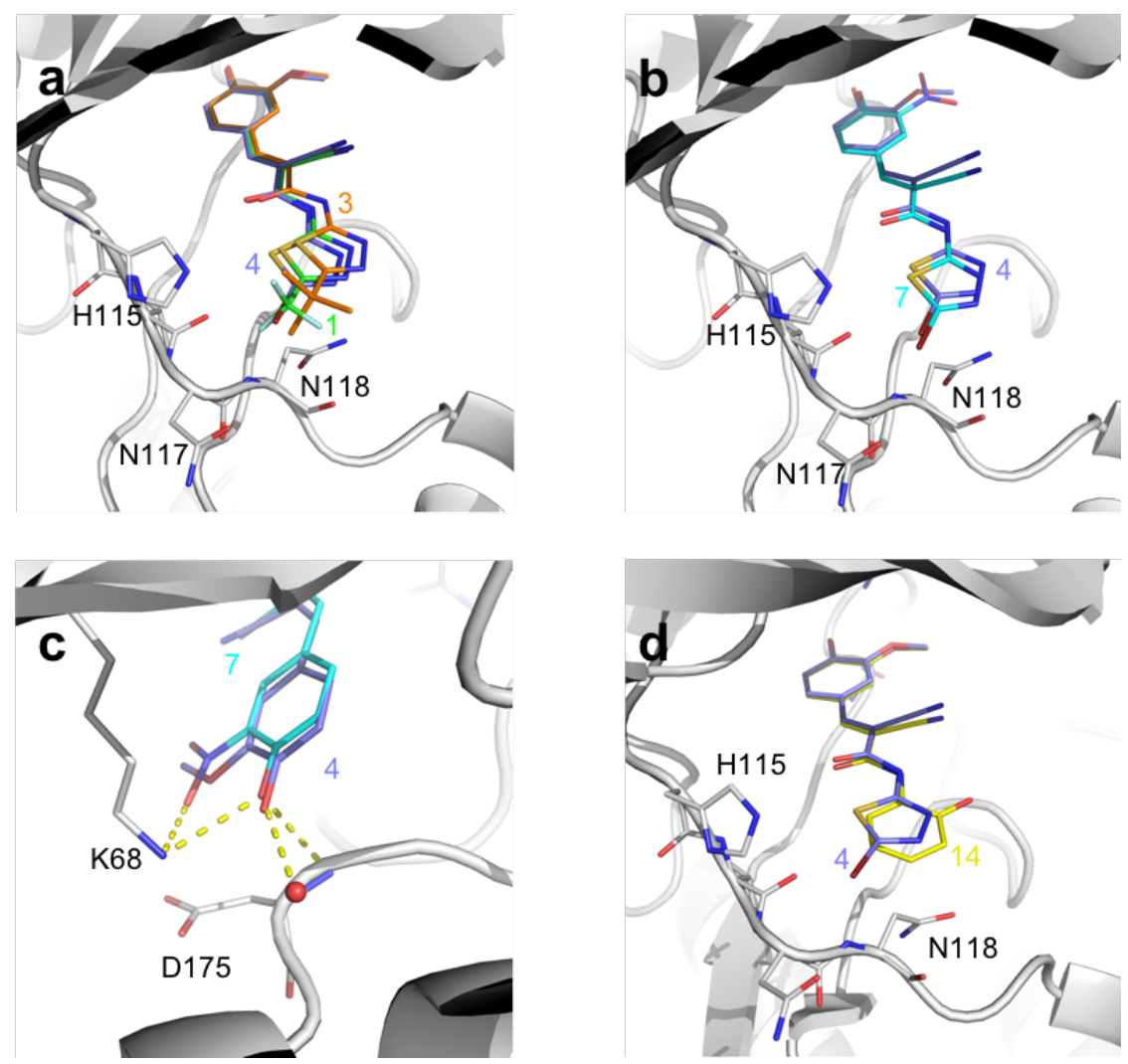

Figure 2. Comparison of binding modes for compounds 1 (green), $\mathbf{3}$ (orange), $\mathbf{4}$ (purple), $\mathbf{7}$ (cyan) and 14 (yellow). a) Tert-butyl-thiadiazole (3) moves away from the hinge region with respect to compound 1, bromo-thiadiazole (4) moves closer. b) The nitrophenol moiety causes the bromothiadiazole of compound 7 to protrude more on the top of the hinge region with respect to the bromothiadiazole of compound $\mathbf{4}$ holding a guaiacol group on the opposite side. c) The nitrophenolic headgroup of compound 7 interacts with CK2 $\alpha$ similarly to what observed in compound 4. d) The ohydroxybenzene ring in compound 14 exposes the phenolic oxygen to the solvent.

We then combined the best hinge-contacting bromo-thiadiazole tail with a $o$-bromophenolic headgroup, with the aim of increasing the acidity of the phenolic oxygen (predicted pKa 7.2) with a small and neutral substituent. Compound 8 shows a modest $\mathrm{IC}_{50}$, being significantly worse than both compounds 4 and $\mathbf{7}$. Compounds $\mathbf{9 - 1 1}$, bearing an aniline ring instead of the guaiacol group of $\mathbf{1}$ and variably substituted on the thiadiazole ring, failed to bind to the kinase when tested by X-ray crystallography, confirming the fundamental role held by acidic groups in complementing the basic region of the CK2 pocket.

Finally, we explored the possibility of substituting the polar thiadiazole 5-member ring with a significantly different benzene ring. A substructure search on the ZINC15 database was performed 
with the 2-cyano-3-(4-hydroxy-3-methoxyphenyl)-N-phenylprop-2-enamide scaffold. The very diverse compounds 12-14 were selected.

Compound 12 bears a 2-chloro-5-trifluoromethyl-benzene ring, which should reproduce the interactions observed in compound $\mathbf{1}$ via its trifluoromethyl group. The compound was not fully soluble at the millimolar concentration needed for the soaking procedure and, although unambiguously present in the CK2 $\alpha$ pocket, its occupancy in the crystallographic structure was limited (data not shown). The measured I $\mathrm{C}_{50}$ towards $\mathrm{CK} 2$ was $0.37 \mu \mathrm{M}$.

Compound 13 bears a $p$-nitrobenzene ring; the nitro group was selected as a possible new specificity determinant to locate in between Arg43 side chain and the hinge downstream to the previously explored region. No extra electron density was present in the CK2 $\alpha$ crystal soaked with compound 13, which then failed to bind to the kinase.

Compound $\mathbf{1 4}$ has a o-hydroxybenzene ring to look for hydrogen bonds with main chain atoms of the hinge region, interactions common in various CK2 inhibitors. The crystal structure shows that the o-hydroxyl group points instead towards the solvent, avoiding the higher energy conformation with the phenolic and the amidic oxygens pointing in the same direction (Fig. 2d).

\section{Target specificity of selected 2-cyano-2-propenamide compounds}

The 2-cyano-2-propenamide compounds here reported are the first CK2 inhibitors protruding from the active site on the top of the hinge region and in direct contact with amino acidic side chains from this region. The peculiar binding mode should define a remarkable selectivity for these compounds. First, compounds 1,4 and $\mathbf{7}$ were tested on the tetrameric CK2 $\alpha_{2} \beta_{2}$ holoenzyme by kinase assay, obtaining very similar $I C_{50}$ as those reported in Table 1 for the $\alpha$ subunit. Then, the most potent compound 4 was tested on a panel of 320 kinases. Only CK $2 \alpha$ and its paralogue CK $2 \alpha^{\prime}$ were inhibited by more than $50 \%$ when tested at $1 \mu \mathrm{M}(\mathrm{SI})$. 
CK2 has a flexible hinge/ $\alpha \mathrm{D}$ region which can assume two different structural arrangements: an open conformation which is extremely rare among protein kinases [19], and a closed conformation recapitulating the correctly assembled catalytic spine (C-spine), as seen in the vast majority of the protein kinases [25]. Binding of the compounds here reported is only compatible with the open conformation of the hinge/ $\alpha \mathrm{D}$ region (Fig. 3). Then, direct interactions with side chains of the hinge region, a feature not previously reported for any CK2 inhibitor only contacting main chain atoms of the same region, guarantee additional selectivity. This is evident when comparing compound 4 with the similar tyrphostin AG99 (PDB 6QS5) [26], interacting only with main chain atoms of the hinge region, compatible with both the open and closed hinge conformations, and not selective (Fig. 3a). The ability to target the CK2 open conformation has recently been exploited by the most selective CK2 inhibitor reported to date [27-28]. However, CAM4066 $\left(\mathrm{IC}_{50}=370 \mathrm{nM}\right)$ has a completely different binding mode and, contrary to the inhibitors reported here, targets the $\alpha \mathrm{D}$ but not the hinge region (Fig. 3b).
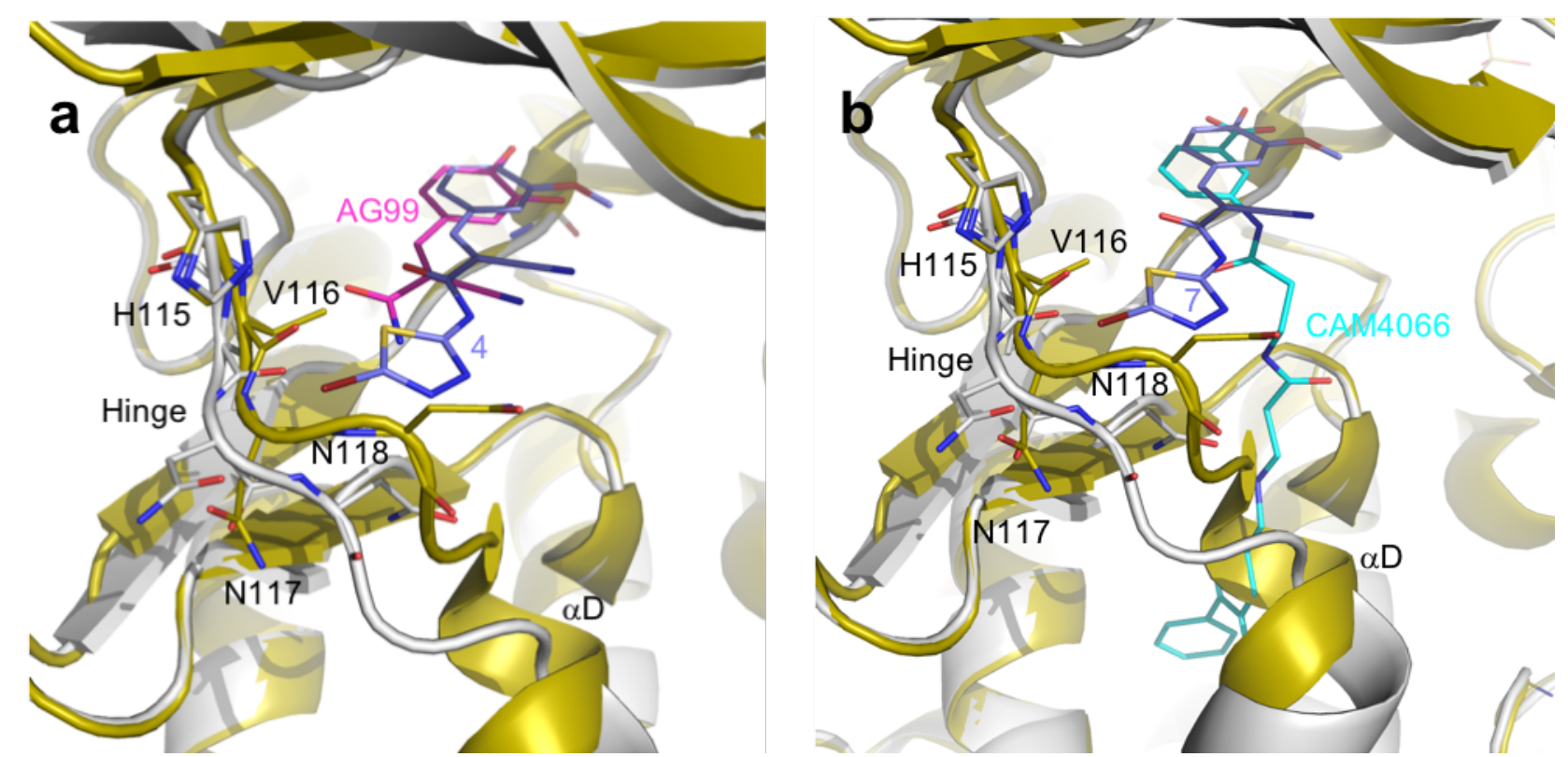

Figure 3. Comparison of binding modes for compound 4 (purple), tyrphostin AG99 (magenta) and CAM4066 (cyan). a) Tyrphostin AG99 interacts with main chain atoms of the hinge region, while compound $\mathbf{4}$ is also in contact with side chains atoms. Binding of compound $\mathbf{4}$ is only compatible with the peculiar open hinge conformation. b) Compounds $\mathbf{4}$ and CAM4066 are compatible with CK2 open conformation (white) but not with the closed conformation (yellow). Compound 4 discriminates the two conformations at the level of the hinge region, while CAM4066 clashes with a closed $\alpha D$ helix. 


\section{Cellular activity of selected 2-cyano-2-propenamide compounds}

The more promising molecules were analyzed for their efficacy in cells, with special focus on the best inhibitor, compound $\mathbf{4}$, in comparison to compound $\mathbf{1}$. We treated Jurkat cells, a human leukemia T-cell line that, since the beginning [29], has been frequently exploited to understand the potential of targeting CK2 in tumor cells. We first assessed whether cells treated with the inhibitors displayed a reduced activity of endocellular CK2. This was confirmed by the analysis of the CK2dependent phospho-site Akt Ser129 [30]: as shown in Fig. 4a, while the amount of the kinase remained intact in treated cells, the Akt phosphorylation level decreased in response to both compound $\mathbf{1}$ and compound $\mathbf{4}$. Since cell death is expected to occur in tumor cells whenever CK2 is inhibited [12], we measured the effect of the compounds on Jurkat cell viability. Results are shown in Fig. 4b, where also the effect of compound $\mathbf{7}$ is reported: all the inhibitors turned out to significantly reduce cell viability in a dose-dependent manner. The $\mathrm{DC}_{50}$ values (concentrations inducing $50 \%$ of cell death compared to vehicle-treated cells in $24 \mathrm{~h}$ ) indicate very similar efficacies for compounds $\mathbf{1}$ and $\mathbf{4}$, while compound $\mathbf{7}$ is less effective. The lower cellular activity of compound 7 can be ascribed to a reduced membrane permeability caused by the negative charge of the $o$ nitrophenolate ring.

a

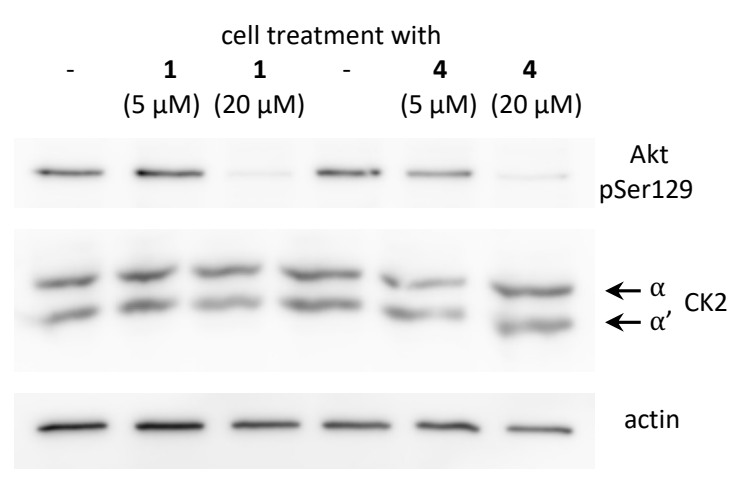

b

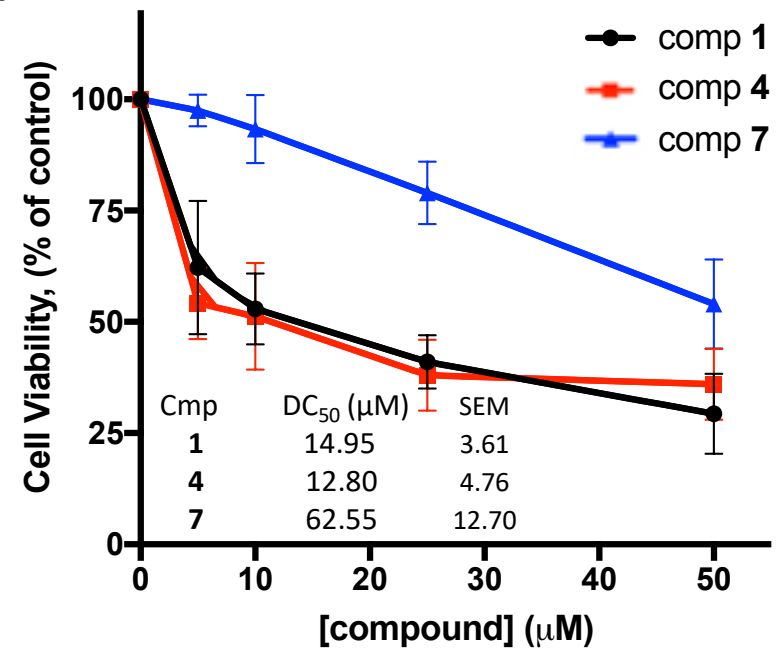

Figure 4. Effects of compounds in cells. A) Jurkat cells were treated with the indicated compounds for $16 \mathrm{~h} .20 \mu \mathrm{g}$ of proteins from total lysate were analysed by WB with anti phospho-Ser129 Akt antibody (upper), or an antibody recognizing both CK2 catalytic subunit $\alpha$ and $\alpha^{\prime}$ (middle). $5 \mu \mathrm{g}$ proteins were analysed for $\beta$-actin (lower WB), as 
loading control. Representative WB of three independent experiments are shown. b) Jurkat cells were treated for $24 \mathrm{~h}$ with increasing concentrations of the indicated compounds. Cell viability was assessed by the MTT method, and is shown as \% of vehicle-treated (Control) cells. On the right, the calculated $\mathrm{DC}_{50}$ values are reported (mean values \pm SEM of at least four experiments).

\section{CONCLUSIONS}

The structural bases for the binding mode of SRPIN803 to CK2 and its reported specificity [16] were puzzling. Its co-crystallization with CK2 $\alpha$ identified a chemical structure different from the one reported (compound 1). De novo synthesis of SRPIN803 also resulted in compound 1. Based on our characterization of compound 1 and SRPIN803, and on previous reports describing very similar discrepancies, in a very similar molecular context, between the expected and the obtained synthetic products [20-21], we conclude that SRPIN803 has indeed the chemical structure of compound 1. Moreover, very similar biologically active compounds based on the 6-methylene-5-imino-1,3,4thiadiazolopyrimidin-7-one scaffold have been described, which are commercially available or obtained through a very similar synthetic scheme (examples in [31-32]). Chemical formulas for these compounds, as well as for the commercially available SRPIN803, could have been similarly misinterpreted.

The crystallographic structure of compound 1 in complex with CK2 $\alpha$ revealed that the observed specificity relies on the exploration of the CK2 open hinge conformation, particularly sporadic in other kinases, combined with interactions with side chains from the same region. Most of the synthesized derivatives of compound 1 showed increased inhibition of CK2 $\alpha$. The most potent compound 4 retained significant selectivity when tested on a panel of 320 kinases, only inhibiting CK2 $\alpha$ and CK $2 \alpha^{\prime}$ by more than $50 \%$ at $1 \mu \mathrm{M}$ concentration. Compound 4 is cell permeable, inhibits endocellular CK2 and significantly reduces Jurkat cells viability. 
Compounds 4, in comparison with the clinical-grade CX-4945 inhibitor and despite the lower potency in kinase assay ( $\mathrm{IC}_{50} 280 \mathrm{nM}$ and $1 \mathrm{nM}$, respectively), shows a similar efficacy in the cellular context: the $12.8 \mu \mathrm{M} \mathrm{DC}_{50}$ that we measured in a $24 \mathrm{~h}$-assay for compound 4 is not far from the 2.5 $\mu \mathrm{M}$ value reported for CX-4945 in a 4-days assay [33]. Moreover, the superiority of compound 4 is also highlighted by its higher selectivity, considering that CX-4945 inhibits at least other 12 kinases in the low nanomolar range [27, 33-35].

\section{MATERIALS AND METHODS}

\section{Chemical syntheses and characterization}

Details of synthetic methods for compounds 1-11 are reported in the Supplementary Materials. ${ }^{1} \mathrm{H}$ and ${ }^{13} \mathrm{C}\left\{{ }^{1} \mathrm{H}\right\}$ NMR spectra were recorded on a Bruker Avance III $400 \mathrm{MHz}$ spectrometer and on a Bruker AMX $300 \mathrm{MHz}$ spectrometer. High resolution mass spectra (HRMS) were recorded on an ESITOF Mariner from Applied Biosystems using electrospray ionization (ESI). The purity profile of the compounds was assayed by HPLC using a Varian Pro-Star system equipped with a Biorad 1706 UVVIS detector and an Agilent C-18 column $(5 \mu \mathrm{m}, 4.6 \times 150 \mathrm{~mm})$. IR spectra were recorded on a PerkinElmer - Spectrum BX FTIR Spectrometer (compound 1) or on a Bruker Equinox 55 FTIR equipped with a single reflection diamond Specac's Golden Gate ${ }^{\mathrm{TM}}$ ATR (compounds 2-11).

Compounds 12-14 were purchased from VITAS-M Laboratory.

All compounds were negative as PAINS or aggregators when tested on dedicated web servers [3637].

\section{Protein production}

The expression of human CK2 $\alpha$ and $\beta$ subunits was induced in E. coli BL21-DE3 with 0,5 mM IPTG for 4 hours at $30^{\circ} \mathrm{C}$. CK2 $\alpha$ pellet mixed with an equal amount of pellet expressing $\beta$ subunit was 
resuspended in buffer $\mathrm{A}$ (50 mM Tris- $\mathrm{HCl}$ pH 8.5 and $7 \mathrm{mM}$ 2-Mercaptoethanol). After sonication and centrifugation, the supernatant was adjusted at the salt concentration of $0,4 \mathrm{M} \mathrm{NaCl}$ and loaded on Heparin-Sepharose ( $5 \mathrm{ml}$, GE Healthcare). The column was eluted with a linear gradient from 0.4 to $1 \mathrm{M} \mathrm{NaCl}$ in buffer $A$. Holoenzyme $\alpha_{2} \beta_{2}$ was purified to homogeneity by size-exclusion chromatography on Superdex 200 (26/60, GE Healthcare) in buffer A + 0,5 M NaCl. The fractions containing the purified holoenzyme were pooled and dialyzed for 4 hours against $25 \mathrm{mM} \mathrm{Tris-HCl}$ $\mathrm{pH} 7,5$ and $50 \%$ glycerol and stored at $-80^{\circ} \mathrm{C}$. The same protocol was applied to purify CK2 $\alpha$ used in activity assays.

CK2 $\alpha$ for crystallization purposes was produced as previously reported [38]. Briefly, after expression in E. coli BL21-DE3, human CK2 $\alpha$ (aa. 1-336) was purified by sequential affinity, anion exchange and size-exclusion chromatography (HiTrap Heparin, MonoQ and Superdex 75). Protein was concentrated to $10 \mathrm{mg} / \mathrm{ml}$ and frozen in liquid nitrogen.

\section{Crystallization and structure solution}

Crystals of apo CK2 and binding of inhibitors in its ATP pocket were obtained as previously described [39]. Briefly, CK2 crystals were first obtained by vapor diffusion sitting drop and then soaked in the cryoprotective solution containing $5 \mathrm{mM}$ inhibitor in 1\% DMSO. Diffraction data were collected at the Elettra Synchrotron Light Source (Trieste, Italy), XRD1 beamline and at the ESRF (Grenoble, France), beamlines ID30-A1 and ID30-A3. Data were processed with XDS [40] and Aimless [41]; structures were solved by molecular replacement with Phaser [42] using PDB 4KWP [43] as a search model. Initial models were refined alternating cycles of automatic refinement with Phenix [44] and manual model building with COOT [45].

Data collection and refinement statistics are reported in Table S1. 
In vitro CK2 activity assay

Recombinant a CK2 (20-50 ng) was incubated with $0.1 \mathrm{mM}$ synthetic peptide substrate RRRADDSDDDDD (CK2-tide) in a phosphorylation buffer containing $50 \mathrm{mM} \mathrm{Tris-HCl} \mathrm{pH} \mathrm{7.5,} 10 \mathrm{mM}$ $\mathrm{MgCl}_{2}, 20 \mu \mathrm{M}\left[\gamma^{-33} \mathrm{P}\right]$ ATP $(1000-2000 \mathrm{cpm} / \mathrm{pmol})$, in a final volume of $30 \mu \mathrm{l}$, with increasing concentrations of each inhibitor. Controls were performed in the absence of any inhibitor, but with the addition of the solvent DMSO $(3 \%, v / v)$, which was ineffective on CK2 activity. Reactions were performed at $30{ }^{\circ} \mathrm{C}$ for $10 \mathrm{~min}$, and stopped by sample absorption on phospho-cellulose papers. Papers were washed three times with $75 \mathrm{mM}$ phosphoric acid and counted in a scintillation counter (Perkin Elmer). The same protocol was applied for the activity of recombinant $\alpha_{2} \beta_{2}$ CK2, except for the addition of $0.1 \mathrm{M} \mathrm{NaCl}$ in the phosphorylation assay. Results were analysed by GraphPad Prism 7.0a software, for the calculation of the $\mathrm{IC}_{50}$ (concentrations inducing $50 \%$ inhibition).

\section{Kinase panel}

The activity of compound 4 was tested at $1 \mu \mathrm{M}$ in duplicate on a 320 protein kinases panel at ProQinase GmbH (Freiburg - Germany, https://www.proqinase.com). A radiometric protein kinase assay ( ${ }^{33}$ PanQinase ${ }^{\circledR}$ Activity Assay) was used for measuring the kinase activities [46].

\section{Cell culture, treatments and lysis}

Jurkat cells (human T lymphoblastoid cell line) were cultured in an atmosphere containing $5 \% \mathrm{CO}_{2}$, maintained in RPMI 1640 medium (Sigma), supplemented with 10\% (v/v) fetal calf serum (FCS), $2 \mathrm{mM}$ L-glutamine, $100 \mathrm{U} / \mathrm{mL}$ penicillin, and $100 \mathrm{mg} / \mathrm{mL}$ streptomycin. Cell treatments with inhibitors were performed in the culture medium, but with $1 \%$ (v/v) FCS. Control cells were treated with equal amounts of the inhibitor solvent (DMSO), which never exceeded $1 \%(\mathrm{v} / \mathrm{v})$. For lysate preparation, 
cells were lysed as described in [47]. Protein concentration was determined by the Bradford method.

\section{Cell viability assay}

Cell viability was detected by means of MTT (3-(4,5-dimethylthiazol-2-yl)-3,5-diphenyltriazolium bromide) reagent: cells $\left(10^{5}\right.$ cells $\left./ 100 \mu \mathrm{l}\right)$ were incubated for $24 \mathrm{~h}$ in a 96 -well plate under the indicated conditions. $1 \mathrm{~h}$ before the end of the incubation, $10 \mu \mathrm{l}$ of MTT solution $(5 \mathrm{mg} / \mathrm{ml}$ in phosphate buffered saline) were added to each well. Incubations were stopped by addition of $20 \mu l$ of lysis solution at $\mathrm{pH} 4.7$, as described elsewhere [47]. Plates were read for OD at $\lambda 590 \mathrm{~nm}$, in a Titertek Multiskan Plus plate reader (Flow Laboratories). DC 50 values (concentrations inducing $50 \%$ cell death in $24 \mathrm{~h}$ ) were calculated by analysing the results with GraphPad Prism 7.0a software.

\section{Endocellular CK2 activity assay}

Endocellular CK2 activity was evaluated by assessing the phosphorylation state of the CK2 substrate Akt phosphor-Ser129 (Abcam) as in [48]. For this purpose, equal amounts of proteins from treated cells were loaded on 11\% SDS-PAGE, blotted on Immobilon-P membranes (Millipore), processed in Western blot (WB), and detected by chemiluminescence. Endocellular CK2 amount was assessed by WB with an antibody recognizing the two catalytic isoforms $\alpha$ and $\alpha^{\prime}$ (Biorad Laboratories). $\beta$-actin antibody, used for normalization, was from Sigma. Quantitation of the signal was obtained by chemiluminescence detection on a Kodak Image Station 440MMPRO and analysis with the Kodak 1DImage software.

\section{ASSOCIATED CONTENT}


The Supporting Information contains further experimental details, tables and figures, as consecutively outlined. Protein Crystallography: collection and refinement statistics, electron density maps, comparison with SRPIN803 docking pose. Small molecule crystallography: methods, results and collection and refinement statistics. Kinase assay: $\mathrm{IC}_{50}$ curves. Kinase panel profiling. Synthetic methods, ${ }^{1} \mathrm{H}-\mathrm{NMR},{ }^{13} \mathrm{C}-\mathrm{NMR}$, IR and HPLC traces for compounds 1-11.

\section{DECLARATION OF INTEREST}

The authors declare no competing financial interest.

\section{ACKNOWLEDGMENTS}

We thank Dr. Massimo Bellanda and Prof. Danilo Pedron (University of Padua, Italy) for their help with NMR and IR spectra acquisition and analysis. We are grateful to the staff of the XRD1 beamline at Elettra (Trieste, Italy) and of the ID30-A1 and ID30-A3 beamlines at ESRF (Grenoble, France), for on-site assistance. G.L. is supported by the Associazione Italiana per la Ricerca sul Cancro (AIRC “MFAG 2017”, code 19882). We are grateful to Prof. Masatoshi Hagiwara (Kyoto University, Japan) for providing us with SRPIN803.

\section{Accession Codes}

Structures were deposited to the PDB with accession numbers 6RB1 (cmp 1), 6RCM (cmp 3), 6RFE (cmp 4), 6RFF (cmp 7) and 6RCB (cmp 14). Authors will release the atomic coordinates and experimental data upon article publication.

\section{References}

1. Nuñez de Villavicencio-Diaz, T., Rabalski, A. J., and Litchfield D.W. (2017) Protein Kinase CK2: Intricate Relationships within Regulatory Cellular Networks. Pharmaceuticals 10, pii: E27. 
2. Pinna, L. A. (2003) The raison d'etre of constitutively active protein kinases: The lesson of CK2. Acc. Chem. Res. 36, 378-384.

3. Lolli, G., Pinna, L. A., and Battistutta, R. (2012) Structural determinants of protein kinase CK2 regulation by autoinhibitory polymerization. ACS Chem. Biol. 7, 1158-1163.

4. Lolli, G., Ranchio, A., and Battistutta, R. (2014) Active form of the protein kinase CK2 $\alpha 2 \beta 2$ holoenzyme is a strong complex with symmetric architecture. ACS Chem. Biol. 9, 366-371.

5. Lolli, G., Naressi, D., Sarno, S., and Battistutta, R. (2017) Characterization of the oligomeric states of the CK2 $\alpha_{2} \beta_{2}$ holoenzyme in solution. Biochem. J. 474, 2405-2416.

6. Chua, M. M. J., Lee, M., and Dominguez, I. (2017) Cancer-type dependent expression of CK2 transcripts. PLoS One 12, e0188854.

7. Piazza, F., Manni, S., Ruzzene, M., Pinna, L. A., Gurrieri, C., and Semenzato, G. (2012) Protein kinase CK2 in hematologic malignancies: reliance on a pivotal cell survival regulator by oncogenic signaling pathways. Leukemia 26, 1174-1179.

8. Kramerov, A. A., Saghizadeh, M., Caballero, S., Shaw, L. C., Li Calzi, S., Bretner, M., Montenarh, M., Pinna, L. A., Grant, M. B., and Ljubimov, A. V. (2008) Inhibition of protein kinase CK2 suppresses angiogenesis and hematopoietic stem cell recruitment to retinal neovascularization sites. Mol. Cell. Biochem. 316, 177-186.

9. Di Maira, G., Brustolon, F., Bertacchini, J., Tosoni, K., Marmiroli, S., Pinna, L. A., and Ruzzene, M. (2007) Pharmacological inhibition of protein kinase CK2 reverts the multidrug resistance phenotype of a CEM cell line characterized by high CK2 level. Oncogene 26, 6915-6926.

10. Ahmad, K. A., Wang, G., Unger, G., Slaton, J., and Ahmed, K. (2008) Protein kinase CK2: A key suppressor of apoptosis. Adv.Enzyme Regul. 48, 179-187. 
11. Wang, G., Unger, G., Ahmad, K. A., Slaton, J. W., and Ahmed, K. (2005) Downregulation of CK2 induces apoptosis in cancer cells: A potential approach to cancer therapy. Mol. Cell. Biochem. 274, 77-84.

12. Ruzzene, M., and Pinna, L. A. (2010) Addiction to protein kinase CK2: a common denominator of diverse cancer cells? Biochim. Biophys. Acta 1804, 499-504.

13. Battistutta, R., Cozza, G., Pierre, F., Papinutto, E., Lolli, G., Sarno, S., O'Brien, S. E., SiddiquiJain, A., Haddach, M., Anderes, K., Ryckman, D. M., Meggio, F., and Pinna, L. A. (2011) Unprecedented selectivity and structural determinants of a new class of protein kinase CK2 inhibitors in clinical trials for the treatment of cancer. Biochemistry 50, 8478-8488.

14. https://www.accessdata.fda.gov/scripts/opdlisting/oopd/detailedIndex.cfm?cfgridkey=548 516

15. clinicaltrials.gov

16. Morooka, S., Hoshina, M., Kii, I., Okabe, T., Kojima, H., Inoue, N., Okuno, Y., Denawa, M., Yoshida, S., Fukuhara, J., Ninomiya, K., Ikura, T., Furuya, T., Nagano, T., Noda, K., Ishida, S., Hosoya, T., Ito, N., Yoshimura, N., and Hagiwara, M. (2015) Identification of a Dual Inhibitor of SRPK1 and CK2 That Attenuates Pathological Angiogenesis of Macular Degeneration in Mice. Mol. Pharmacol. 88, 316-325.

17. Bullock N. and Oltean S. (2017) The many faces of SRPK1. J. Pathol. 241, 437-440.

18. Amin, E. M., Oltean, S., Hua, J., Gammons, M. V., Hamdollah-Zadeh, M., Welsh, G. I., Cheung, M. K., Ni, L., Kase, S., Rennel, E. S., Symonds, K. E., Nowak, D. G., Royer-Pokora, B., Saleem, M. A., Hagiwara, M., Schumacher, V. A., Harper, S. J., Hinton, D. R., Bates, D. O., and Ladomery, M. R. (2011) WT1 Mutants Reveal SRPK1 to Be a Downstream Angiogenesis Target by Altering VEGF Splicing. Cancer. Cell 20, 768-780. 
19. Battistutta, R., and Lolli, G. (2011) Structural and functional determinants of protein kinase CK2 $\alpha$ : facts and open questions. Mol. Cell. Biochem. 356, 67-73.

20. Busch, B. B., Stevens, W. C. Jr., Martin, R., Ordentlich, P., Zhou, S., Sapp, D. W., Horlick, R. A., and Mohan, R. (2004) Identification of a selective inverse agonist for the orphan nuclear receptor estrogen-related receptor alpha. J. Med. Chem. 47, 5593-5596.

21. Liu, H., Wu, R., Sun, Y., Ye, Y., Chen, J., Luo, X., Shen, X., and Liu, H. (2014) Identification of novel thiadiazoloacrylamide analogues as inhibitors of dengue-2 virus NS2B/NS3 protease. Bioorg. Med. Chem. 22, 6344-6352.

22. Battistutta, R., Mazzorana, M., Cendron, L., Bortolato, A., Sarno, S., Kazimierczuk, Z., Zanotti, G., Moro, S., and Pinna L. A. (2007) The ATP-binding site of protein kinase CK2 holds a positive electrostatic area and conserved water molecules. Chembiochem 8, 1804-1809.

\section{3. https://chemicalize.com}

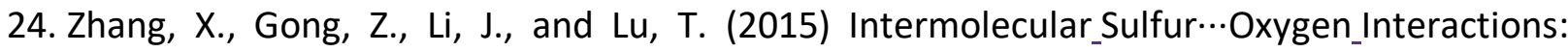
Theoretical and Statistical Investigations. J. Chem. Inf. Model. 55, 2138-2153.

25. Kornev, A. P., and Taylor, S. S. (2010) Defining the conserved internal architecture of a protein kinase. Biochim. Biophys. Acta 1804, 440-444.

26. Lolli, G., Cozza, G., Mazzorana, M., Tibaldi, E., Cesaro, L., Donella-Deana, A., Meggio, F., Venerando, A., Franchin, C., Sarno, S., Battistutta, R., and Pinna, L.A. (2012) Inhibition of protein kinase CK2 by flavonoids and tyrphostins. A structural insight. Biochemistry 51, 60976107.

27. Brear, P., De Fusco, C., Georgiou, K. H., Francis-Newton, N. J., Stubbs, C. J., Sore, H. F., Venkitaraman, A. R., Abell, C., Spring, D. R., and Hyvönen, M. (2016) Specific inhibition of CK2 $\alpha$ from an anchor outside the active site. Chem. Sci. 7, 6839-6845. 
28. De Fusco, C., Brear, P., legre, J., Georgiou, K. H., Sore, H. F., Hyvönen, M., and Spring, D. R. (2017) A fragment-based approach leading to the discovery of a novel binding site and the selective CK2 inhibitor CAM4066. Bioorg. Med. Chem. 25, 3471-3482.

29. Ruzzene, M., Penzo, D., and Pinna, L. A. (2002) Protein kinase CK2 inhibitor 4,5,6,7tetrabromobenzotriazole (TBB) induces apoptosis and caspase-dependent degradation of haematopoietic lineage cell-specific protein 1 (HS1) in Jurkat cells. Biochem. J. 364, 41-47.

30. Di Maira, G., Salvi, M., Arrigoni, G., Marin, O., Sarno, S., Brustolon, F., Pinna, L. A., and Ruzzene, M. (2005) Protein kinase CK2 phosphorylates and upregulates Akt/PKB. Cell Death Differ. 12, 668-677.

31. Paudel, A., Kaneko, K., Watanabe, A., Matsunaga, S., Kanai, M., Hamamoto, H., and Sekimizu, K. (2013) Structure-activity relationship study of novel iminothiadiazolopyrimidinone antimicrobial agents. J. Antibiot. 66, 663-667.

32. Park, H., Lee, S., and Hong, S. (2012) Discovery of MEK/PI3K dual inhibitor via structurebased virtual screening. Bioorg. Med. Chem. Lett. 22, 4946-4950.

33. Pierre, F., Chua, P. C., O'Brien, S. E., Siddiqui-Jain, A., Bourbon, P., Haddach, M., Michaux, J., Nagasawa, J., Schwaebe, M. K., Stefan, E., Vialettes, A., Whitten, J. P., Chen, T. K., Darjania, L., Stansfield, R., Anderes, K., Bliesath, J., Drygin, D., Ho, C., Omori, M., Proffitt, C., Streiner, N., Trent, K., Rice, W. G., and Ryckman, D. M. (2011) Discovery and SAR of 5-(3chlorophenylamino)benzo[c][2,6]naphthyridine-8-carboxylic acid (CX-4945), the first clinical stage inhibitor of protein kinase CK2 for the treatment of cancer. J. Med. Chem. 54, 635-654.

34. Kim, H., Lee, K. S., Kim, A. K., Choi, M., Choi, K., Kang, M., Chi, S. W., Lee, M. S., Lee, J. S., Lee, S. Y., Song, W. J., Yu, K., and Cho, S. (2016) A chemical with proven clinical safety rescues Down-syndrome-related phenotypes in through DYRK1A inhibition. Dis. Model Mech. 9, 839848. 
35. Kim, H., Choi, K., Kang, H., Lee, S. Y., Chi, S. W., Lee, M. S., Song, J., Im, D., Choi, Y., and Cho, S. (2014) Identification of a novel function of CX-4945 as a splicing regulator. PLoS One 9, e94978.

36. Baell J. B., and Holloway, G. A. (2010) New Substructure Filters for Removal of Pan Assay Interference Compounds (PAINS) from Screening Libraries and for Their Exclusion in Bioassays. J. Med. Chem., 53, 2719-2740

37. Irwin, J. J., Duan, D., Torosyan, H., Doak, A. K., Ziebart, K. T., Sterling, T., Tumanian, G., and Shoichet, B. K. (2015) An Aggregation Advisor for Ligand Discovery. J. Med. Chem. 58, 70767087.

38. Papinutto, E., Ranchio, A., Lolli, G., Pinna, L. A., and Battistutta, R. (2012) Structural and functional analysis of the flexible regions of the catalytic $\alpha$-subunit of protein kinase CK2. J. Struct. Biol. 177, 382-391.

39. Battistutta, R., and Lolli, G. (2019) Inhibitory Properties of ATP-Competitive Coumestrol and Boldine Are Correlated to Different Modulations of CK2 Flexibility. J. Nat. Prod. 82, 10141018.

40. Kabsch, W. (2010) XDS. Acta Crystallogr. D Biol. Crystallogr. 66, 125-132.

41. Evans, P. R., and Murshudov, G. N. (2013) How good are my data and what is the resolution? Acta Crystallogr. D Biol. Crystallogr. 69, 1204-1214.

42. McCoy, A. J., Grosse-Kunstleve, R. W., Adams, P. D., Winn, M. D., Storoni, L. C., and Read, R. J. (2007) Phaser crystallographic software. J. Appl. Crystallogr. 40, 658-674.

43. Cozza, G., Girardi, C., Ranchio, A., Lolli, G., Sarno, S., Orzeszko, A., Kazimierczuk, Z., Battistutta, R., Ruzzene, M., and Pinna, L. A. (2014) Cell-permeable dual inhibitors of protein kinases CK2 and PIM-1: structural features and pharmacological potential. Cell. Mol. Life Sci. $71,3173-3185$. 
44. Adams, P. D., Afonine, P. V., Bunkóczi, G., Chen, V. B., Davis, I. W., Echols, N., Headd, J. J., Hung, L.-W., Kapral, G. J., Grosse-Kunstleve, R. W., McCoy, A. J., Moriarty, N. W., Oeffner, R., Read, R. J., Richardson, D. C., Richardson, J. S., Terwilliger, T. C., and Zwart, P. H. (2010) PHENIX: a comprehensive Python-based system for macromolecular structure solution. Acta Crystallogr. D Biol. Crystallogr. 66, 213-221.

45. Emsley, P., Lohkamp, B., Scott, W. G., and Cowtan, K. (2010) Features and development of Coot. Acta Crystallogr. D Biol. Crystallogr. 66, 486-501.

46. Horbert, R., Pinchuk, B., Johannes, E., Schlosser, J., Schmidt, D., Cappel, D., Totzke, F., Schächtele, C., and Peifer, C. (2015) Optimization of potent DFG-in inhibitors of platelet derived growth factor receptor $\beta$ (PDGF-R $\beta$ ) guided by water thermodynamics. J. Med. Chem. $58,170-82$.

47. Zanin, S., Borgo, C., Girardi, C., O'Brien, S. E., Miyata, Y., Pinna, L. A., Donella-Deana, A., and Ruzzene, M. (2012) Effects of the CK2 inhibitors CX-4945 and CX-5011 on drug-resistant cells. PLoS One 7, e49193.

48. Di Maira, G., Brustolon, F., Pinna, L. A., and Ruzzene, M. (2009) Dephosphorylation and inactivation of Akt/PKB is counteracted by protein kinase CK2 in HEK 293T cells. Cell. Mol. Life Sci. 66, 3363-3373. 
Table 1: Chemical structures of tested compounds

\begin{tabular}{|c|c|c|c|}
\hline Compound & $\mathbf{R}_{\mathbf{1}}$ & $\mathbf{R}_{\mathbf{2}}$ & $\mathrm{IC}_{50}(\mu \mathrm{M})(\mathrm{SEM})$ \\
\hline 1 & & & $1.37(0.20)$ \\
\hline 2 & & & $0.88(0.44)$ \\
\hline 3 & & & $0.36(0.10)$ \\
\hline 4 & & & $0.28(0.09)$ \\
\hline 5 & & & $0.71(0.28)$ \\
\hline 6 & & & $0.32(0.08)$ \\
\hline 7 & & & $0.37(0.08)$ \\
\hline
\end{tabular}




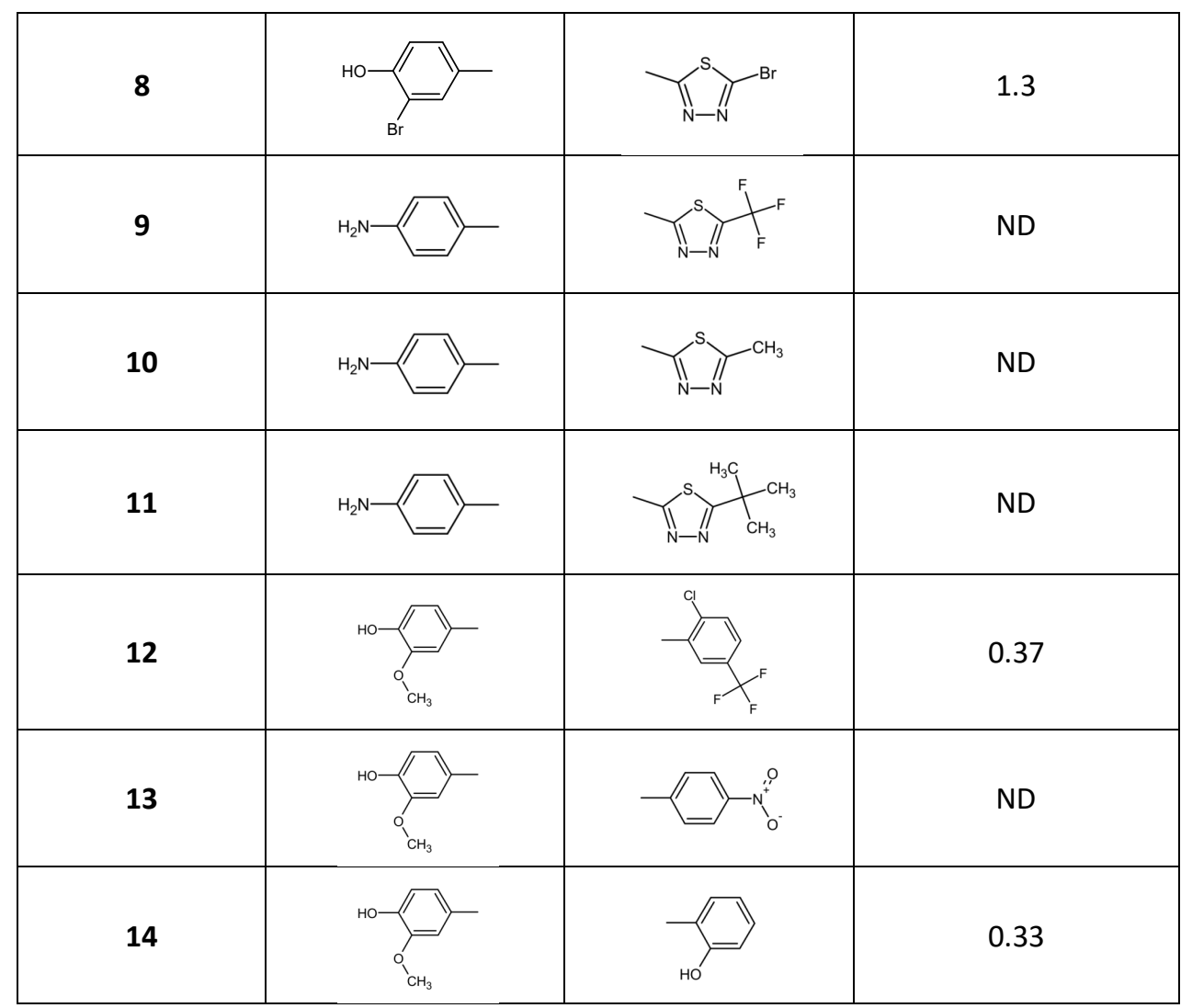

$\mathrm{ND}=$ not determined; compounds failed to bind the kinase in the crystal were not tested in kinase assay

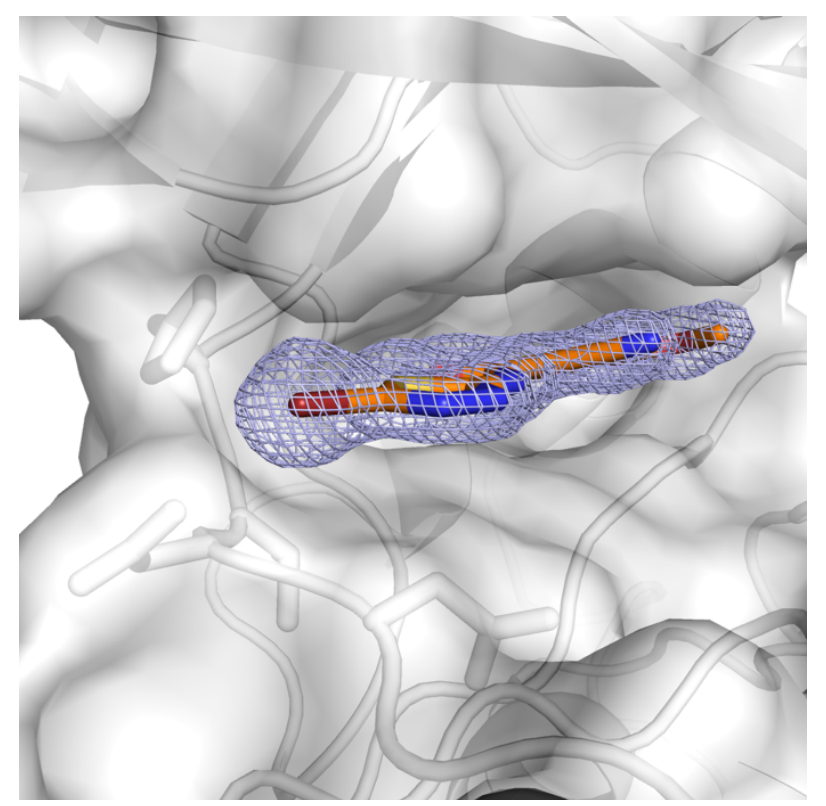

Graphical abstract 